\title{
Building Novel Carbon Allotropes
}

\author{
Calculations indicate that a form of carbon synthesized from pentagonal \\ hydrocarbon molecules could have unusual electrical and mechanical \\ properties. (See Editor's Note.) \\ By Rachel Berkowitz
}

\section{The paper highlighted in this Synopsis has been retracted. For more information, see the retraction note here.}

In diamond, as in other familiar forms of carbon, atoms are bound to one another such that they all have the same electronic structure. Combining carbon atoms so that their electronic configurations vary, however, yields forms with unexpected physical properties, such as magnetism and hardness surpassing even that of diamond. So far, such materials have been created only by treating existing forms with heat and pressure. Now, Yasumaru Fujii and colleagues at the University of Tsukuba, Japan, identify, using quantum-mechanical simulations, a potentially useful material that could be synthesized from the bottom up. The results show that novel, stable carbon allotropes could be custom designed with properties suited to specific applications.

Fujii and his colleagues simulated the assembly of a covalent 3D

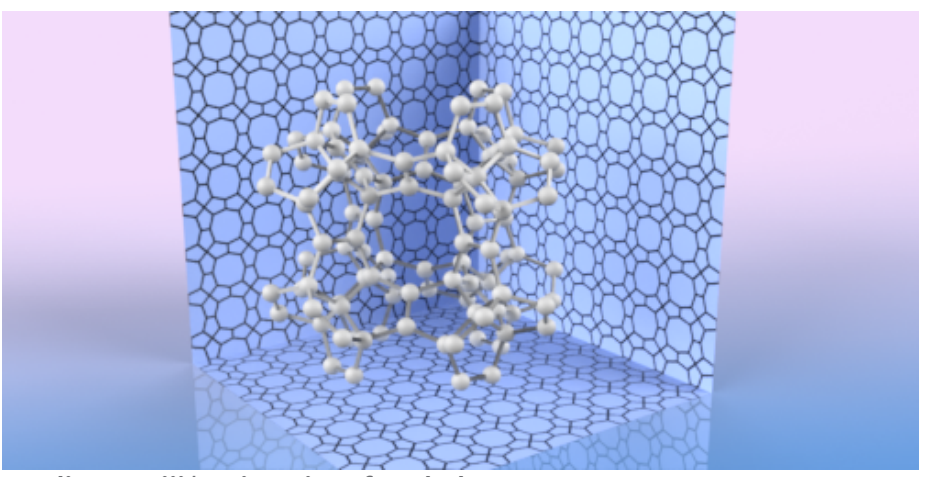

Credit: Y. Fujii/ University of Tsukuba network from a mixture of two hydrocarbon molecules, both containing pentagonal carbon rings. Calculations of the network's total energy and electronic structure confirmed its stability and revealed it to be an indirect semiconductor. Their model also indicated that the material, which they call pentadiamond, has some notable mechanical properties. First, it is nearly as hard as diamond yet is much less dense. Second, it has a negative Poisson's ratio, meaning it becomes thicker when stretched. The researchers attribute the hardness to the combination of electronic configurations present in the structure: while some atoms bind to produce one geometry, others reinforce the network like cross braces in a building. The material's unusual behavior under strain, meanwhile, could be due to its pentagonal components.

The researchers say that pentadiamond and other novel materials could be created easily using conventional copolymer synthesis methods, and they could be useful as reinforcement materials in technologies such as gas storage, chemical engineering, and optoelectronics.

This research is published in Physical Review Letters.

Rachel Berkowitz is a Corresponding Editor for Physics based in Seattle, Washington, and Vancouver, British Columbia. 